\title{
Polymorphisms in the transcription factor NRF2 and forearm vasodilator responses in humans
}

\author{
Ewa D. Marczak ${ }^{a}$, Jacqui Marzeca, Darryl C. Zeldina ${ }^{a}$, Steven R. Kleebergera, Nancy J. \\ Brown $^{c}$, Mias Pretorius ${ }^{d}$, and Craig R. Lee ${ }^{b}$ \\ aDivision of Intramural Research, National Institute of Environmental Health Sciences, National \\ Institutes of Health, Research Triangle Park \\ bDivision of Pharmacotherapy and Experimental Therapeutics, Eshelman School of Pharmacy, \\ University of North Carolina, Chapel Hill, North Carolina \\ 'Departments of Medicine and Pharmacology, Division of Clinical Pharmacology, Vanderbilt \\ University Medical Center, Nashville, Tennessee, USA \\ dDepartment of Anesthesiology, Vanderbilt University Medical Center, Nashville, Tennessee, \\ USA
}

\begin{abstract}
Objective-Oxidative stress is integral to the development of endothelial dysfunction and cardiovascular disease. As NRF2 is a key transcription factor in antioxidant defense, we aimed to determine whether polymorphisms within the promoter region of the gene encoding NRF2 (NFE2L2) would significantly modify vasodilator responses in humans.
\end{abstract}

Methods-Associations between the - 653A/G(rs35652124), - 651G/A (rs6706649), and 617C/A (rs6721961) polymorphisms within the NFE2L2 promoter and vascular function were evaluated in healthy African-American $(n=64)$ and white $(n=184)$ individuals. Forearm blood flow $(\mathrm{FBF})$ was measured by strain-gauge venous occlusion plethysmography at baseline and in response to incremental doses of bradykinin or sodium nitroprusside. Forearm vascular resistance (FVR) was calculated as the mean arterial pressure/FBF.

Results-In African Americans, - $653 G$ variant allele carriers had significantly lower FBF and higher FVR under basal conditions as well as in response to bradykinin or sodium nitroprusside compared with wild-type individuals ( $P<0.05$ for each comparison). In whites, although no significant associations were observed with the $-653 A / G$ genotype, $-617 A$ variant allele carriers had significantly higher FVR at baseline and in response to bradykinin or sodium nitroprusside compared with wild-type individuals ( $P<0.05$ for each comparison). The $-651 G / A$ polymorphism was not associated with vasodilator responses in either racial group.

Conclusion-Polymorphisms within the NFE2L2 promoter were associated with impaired forearm vasodilator responses in an endothelial-independent manner, suggesting an important role of NRF2 in the regulation of vascular function in humans.

(C) 2012 Wolters Kluwer Health | Lippincott Williams \& Wilkins

Correspondence to Craig R. Lee, PharmD, PhD, CB\# 7569, Kerr Hall, Division of Pharmacotherapy and Experimental Therapeutics, UNC Eshelman School of Pharmacy, Chapel Hill, NC 27599-7569, USA, Tel: + 1919843 7673; fax: +1 919 962 0644;

craig_lee@unc.edu.

Ewa D. Marczak and Jacqui Marzec contributed equally to the writing of this article.

Conflicts of interest There are no conflicts of interest.

Supplemental digital content is available for this article. Direct URL citations appear in the printed text and are provided in the HTML and PDF versions of this article on the journal's Website (www.pharmacogeneticsandgenomics.com). 


\section{Keywords}

bradykinin; NFE2L2; NRF2; polymorphism; sodium nitroprusside; vascular function

\section{Introduction}

Endothelial dysfunction is a common and early feature of vascular disease that is manifested by impaired peripheral and coronary artery vasodilator responses, and is present in patients with hypertension, hypercholesterolemia, and diabetes mellitus, and in older patients [1,2]. In particular, reduced vasodilator responses occur early in atherogenesis, before angiographic evidence of disease [3], and is significantly associated with the development of atherosclerosis and clinical events such as myocardial infarction and stroke [4,5]. Oxidative stress is an integral mediator of the development of endothelial dysfunction and consequently the development and progression of atherosclerosis $[1,2]$.

Nuclear factor E2-related factor 2 (NRF2) is a member of the basic leucine zipper (bZip) transcription factors [6]. NRF2 regulates cellular defense against various detrimental stresses including oxidative stress by controlling the basal and inducible expression of antioxidant genes, phase II detoxifying enzymes, transporters, scavenger receptors, chaperone proteins, and transcription factors [7-9]. NRF2 is ubiquitously expressed in the cardiovascular system, where it plays a key role in defense against cytotoxic assault [10,11]. In human aortic endothelial cells, NRF2 overexpression inhibited hydrogen peroxide-induced cytotoxicity, as well as induction of monocyte chemoattractant protein- 1 and vascular cell adhesion molecule- 1 expression and monocyte adhesion in response to the proinflammatory cytokine tumor necrosis factor [12]. NRF2 activation also reversed the dysfunction of endothelial cells induced by hyperglycemia [13]. Moreover, induction of NRF2-dependent antioxidant gene expression in response to oxidized phospholipids was lower in the carotid arteries of $N_{r} 2$ knockout compared with wild-type mice, suggesting a potential antiatherogenic effect of NRF2 [14].

In humans, three single nucleotide polymorphisms within the promoter region of the gene encoding NRF2 (NFE2L2) were identified recently [ - 653A/G(rs35652124), - 651G/A (rs6706649), and - 617C/A (rs6721961)], and each minor allele showed significantly reduced NFE2L2 promoter activity in transfected A549 human alveolar adenocarcinoma cells in vitro [15]. Moreover, $-617 A$ variant allele carriers showed a significantly higher risk of acute lung injury, an oxidative stress-mediated condition [15]. In contrast, the role of NRF2 in the regulation of vascular function in humans remains largely unexplored. Because of the integral role of NRF2 in antioxidant defense, and the impact of oxidative stress on the development and severity of endothelial dysfunction, we hypothesized that functionally relevant polymorphisms within the promoter region of NFE2L2 would significantly modify endothelium-dependent and endothelium-independent vasodilator responses in humans. Consequently, the objective of the present investigation was to evaluate the impact of the $N F E 2 L 2-653 A / G,-651 G / A$, and $-617 C / A$ polymorphisms on forearm vasodilator responses to bradykinin and sodium nitroprusside.

\section{Methods}

\section{Participants}

Healthy volunteers $(n=248)$ who participated in studies that evaluated endotheliumdependent and endothelium-independent forearm vasodilator responses to bradykinin and sodium nitroprusside, respectively, were included [16]. Participants were classified as either African Americans ( $n=64)$ or whites $(n=184)$. Each participant was asked to self-report their 
race and indicate whether all four of their grandparents had self-defined as the same race. If the participants and all four of their grandparents did not self-define as the same race, they were excluded from the study. All participants gave written informed consent and underwent a history, physical examination, laboratory screening, and electrocardiogram. Patients with significant cardiovascular, renal, pulmonary, endocrine, or hematological disease were excluded. All participants were within $30 \%$ of their ideal body weight and none were taking medications, including angiotensin-converting enzyme inhibitors. Pregnancy was an exclusion criterion in women of childbearing potential, and was determined by the measurement of urine $\beta$-human chorionic gonadotropin. The protocol was approved by the Institutional Review Board at each institution and was conducted according to the Declaration of Helsinki.

\section{Phenotype assessment}

Studies were performed in a temperature-controlled room in the morning after an overnight fast, as described previously [16,17]. A 20-G polyurethane catheter was inserted into the brachial artery of the nondominant arm, and arterial catheter patency was maintained by an infusion of $0.9 \%$ sodium chloride at a rate of $1 \mathrm{ml} / \mathrm{min}$. Participants were allowed to rest for $30 \mathrm{~min}$ before baseline measurements and between drug infusions. Heart rate and blood pressure were monitored continuously in the contralateral arm throughout the infusion protocol using an automated blood pressure cuff (GE Medical Systems, Tampa, Florida, USA). Forearm blood flow (FBF) was measured using strain-gauge venous occlusion plethysmography (DE Hokanson, Bellevue, Washington, USA) at baseline and in response to incremental doses of bradykinin (100 and $200 \mathrm{ng} / \mathrm{min}$; Clinalfa AG, Läufelfingen, Switzerland). Sodium nitroprusside (1.6, 3.2, and $6.4 \mu \mathrm{g} / \mathrm{min}$; Gensia Siccor Pharmaceuticals, Irvine, California, USA) was administered to 231 participants as an endothelium-independent control. Each drug dose was infused for 5min, and FBF was measured during the last 2 min of each drug infusion protocol. The sequence of drug administration was randomized to avoid an order effect. FBF is expressed in $\mathrm{ml} / 100 \mathrm{ml}$ of volume of tissue/minute. Forearm vascular resistance (FVR) is calculated as the ratio of the mean arterial pressure/FBF and expressed in $\mathrm{mmHg} / \mathrm{ml} / 100 \mathrm{ml}$ of volume of tissue/minute.

Evaluation of FBF following an intra-arterial infusion of bradykinin and sodium nitroprusside, respectively, is a well-established method to assess endothelial-dependent and endothelial-independent vasodilator responses in humans [1]. Importantly, these peripheral vascular phenotypes correlate with vasodilator responses in coronary arteries and predict the risk of developing cardiovascular disease [5].

\section{Genotyping}

DNA was isolated from whole-blood samples by a modified phenol-chloroform extraction. The $-653 A / G,-651 G / A$, and $-617 C / A$ genotypes were determined by direct sequencing of the -738 to -461 region within the NFE2L2 promoter, as described previously [15]. Initial PCR products were generated with the Epicentre Failsafe system (Epicentre Biotechnologies, Madison, Wisconsin, USA) using $50 \mathrm{ng}$ of genomic DNA, $1 \mu \mathrm{mol} / \mathrm{l}$ each of forward ( $5^{\prime}$-GACCACTCTCCGACCTAAAGG-3 $\left.{ }^{\prime}\right)$ and reverse primer $\left(5^{\prime}\right.$ CGAGATAAAGAGTTGTTTGCGAA- $3^{\prime}$ ), $12.5 \mu$ l of Failsafe buffer E, and $0.25 \mathrm{U}$ of Failsafe enzyme mix. The PCR conditions were as follows: initial denaturation at $95^{\circ} \mathrm{C}$ for 4 min, followed by 35 cycles of $95^{\circ} \mathrm{C}$ for $1 \mathrm{~min}, 56^{\circ} \mathrm{C}$ for $1 \mathrm{~min}, 72^{\circ} \mathrm{C}$ for $1 \mathrm{~min}$, and a final extension at $72^{\circ} \mathrm{C}$ for $8 \mathrm{~min}$. Products were purified using the GenElute PCR cleanup kit (Sigma Chemical Co., St Louis, Missouri, USA) and a portion was run on $3 \%$ agarose gels to confirm amplification. Sequencing reactions were carried out using the Big Dye terminator kit and analyzed on a Perkin Elmer ABI 3100 Automated DNA Sequencer (Applied Biosystems, Foster City, California, USA). Ambiguous samples were verified 
using overlapping reads with an internal primer (5'-CTTTTA

TCTCACTTTACCGCCC- $3^{\prime}$ ). All samples were randomized and genotyped, and the results were read by two independent researchers with $100 \%$ concordance.

\section{Transient transfection reporter gene assay}

The impact of each polymorphism on the activity of the NFE2L2 promoter in endothelial cells, under both basal and stimulated conditions, was assessed using reporter gene assays. As described previously [15], constructs containing the $-653 G,-651 A$, and $-617 A$ variant alleles were generated by cloning the -727 to +131 region of the NFE2L2 promoter into a pGL3 vector upstream of the luciferase reporter gene. Sequence-verified constructs were transiently transfected into human microvascular endothelial cells (HMVEC-L, Lonza Inc., Walkersville, Maryland, USA) and maintained in media supplemented with $10 \%$ fetal bovine serum (Sigma). DNA constructs were transfected using the Amaxa HMVEC-L nucleofector kit (Lonza Inc.) according to the manufacturer's specifications. HMVEC cells grown to 70-80\% confluence on 12-well transwell plates were cotransfected with $0.5 \mu \mathrm{g}$ of the promoter construct, or an empty (promoter-less) pGL3 vector, and $5 \mu \mathrm{g}$ of pRL-TK (renilla) DNA as a transfection efficiency internal control. Twenty-four hours after transfection, cells were incubated with vehicle (medium), bradykinin $(0.01$ or $0.1 \mathrm{mmol} / \mathrm{l})$, sodium nitroprusside $(0.1 \mathrm{mmol} / \mathrm{l})$, or hydrogen peroxide $(0.1 \mathrm{mmol} / \mathrm{l})$ for an additional $24 \mathrm{~h}$ and reporter gene activity was measured using the Promega Dual-Luciferase reporter assay system (Promega Corp., Madison, Wisconsin, USA). The concentrations were selected after the completion of preliminary experiments that aimed to induce a two- to three-fold upregulation in promoter activity (data not shown). Luciferase activity was measured in three independent samples, and experiments were repeated in triplicate.

\section{Statistical analysis}

Race-stratified allele frequencies were calculated and evaluated for deviation from HardyWeinberg Equilibrium (HWE) by comparing the observed and the expected frequencies using the $\chi^{2}$-test. HWE and pairwise linkage disequilibrium (LD) statistics were calculated using Haploview 4.2. Differences in the baseline characteristics among the genotype groups for continuous variables were determined using Student's $t$-test or analysis of variance (ANOVA), where appropriate. A $\chi^{2}$-test was used to compare categorical variables. Variables that were not normally distributed, including FBF and FVR, were log transformed before analysis.

Differences in FBF and FVR were evaluated by general linear model-repeated measures ANOVA in which the within-participant variable was bradykinin or sodium nitroprusside dose and the between-participant variable was genotype group. Because of previously reported racial differences in vasodilator responses in this and other cohorts $[16,18,19]$, all analyses were stratified by race. Genotype frequencies governed the appropriate model to use. An additive mode of inheritance was initially applied for each polymorphism, but was retained only for the $-653 A / G$ polymorphism in whites. Because of the low number of participants homozygous for variant alleles, a dominant mode of inheritance was used in all the other comparisons. In instances where the general linear model-repeated measures ANOVA was statistically significant $(P<0.05)$, a post-hoc Scheffe's test was utilized to evaluate between-group differences at each dose. A $P$-value less than 0.05 was used to determine statistical significance. Data are presented as mean \pm SEM unless otherwise indicated. Statistical analyses were performed using SAS 9.1 for Windows (SAS Institute Inc., Cary, North Carolina, USA).

To minimize the impact of the multiple statistical tests carried out, the false discovery rate $q$ value was estimated using QVALUE for each repeated-measures ANOVA test [20]. The $q$ - 
value is defined as the expected proportion of statistical tests considered significant that are actually false positives. Only $q$-values for statistically significant findings $(P<0.05)$ are presented. Post-hoc power calculations were completed using QUANTO [21].

In the reporter gene experiments, data were expressed relative to the wild-type vehicle (control) group, and differences across genotype and treatment were evaluated using an ANOVA and the Student-Newman-Keuls a posteriori comparisons of means (SigmaPlot 12, Systat Software Inc., Chicago, Illinois, USA). A two-sided $P$-value of less than 0.05 was considered statistically significant.

\section{Results}

\section{Participant characteristics and NFE2L2 genotype distribution}

Race-stratified participant characteristics are presented in Table 1. In comparison with the white participants, the African-American cohort had significantly higher blood pressure, FVR, and BMI, and included significantly more women. All other characteristics were not significantly different between groups.

Race-stratified genotype distributions and allelic frequencies for each polymorphism are presented in Table 2 . The $-653 G$ and $-617 A$ allele frequencies were significantly higher in whites compared with African Americans; however, the $-651 \mathrm{~A}$ allele frequency did not differ between racial groups. The genotype distributions for each polymorphism were in HWE within each racial group ( $P>0.20$ for each comparison). No evidence of pairwise LD was observed between the three polymorphisms in either racial group $\left(I^{2}<0.01\right.$ in African Americans, $r^{2}<0.07$ in whites for all comparisons).

No statistically significant differences in age, sex, cigarette smoking status, BMI, or resting blood pressure were observed across any of the genotype groups (see Supplemental Tables 1, 2, and 3, Supplemental digital content 1, http://links.lww.com/FPC/A482, which provides race-stratified comparisons of participant characteristics by $-653 A / G,-651 G / A$, and $617 C / A$ genotypes, respectively).

\section{NFE2L2 genotype and vasodilator responses to bradykinin and sodium nitroprusside}

An infusion of bradykinin (100 and $200 \mathrm{ng} / \mathrm{min}$ ) and sodium nitroprusside (1.6, 3.2, and 6.4 $\mu \mathrm{g} / \mathrm{min}$ ) caused a dose-dependent increase in FBF and decrease in FVR in both African Americans and whites (Figs 1-3).

\section{$-653 A / G$}

In African Americans, - $653 G$ variant allele carriers had significantly lower baseline FBF $(2.7 \pm 1.1$ vs. $4.1 \pm 1.1 \mathrm{ml} / 100 \mathrm{ml}$ tissue $/ \mathrm{min} ; P<0.001)$ and higher FVR $(31.1 \pm 1.1$ vs. $22.5 \pm 1.1 \mathrm{mmHg} / \mathrm{ml} / 100 \mathrm{ml}$ tissue $/ \mathrm{min} ; P=0.006$ ) compared with wild-type (A/A) individuals, respectively. The vasodilator response to bradykinin was significantly associated with the $-653 A / G$ genotype, such that FBF was significantly lower (Fig. 1a, $P=0.003, q=0.020$ ) and FVR was significantly higher (Fig. $1 \mathrm{~b}, P=0.040, q=0.130$ ) in $-653 G$ variant allele carriers relative to wild-type individuals. Statistically significant associations between the $-653 A / G$ genotype and both FBF (Fig. 1c, $P<0.001, q=0.010$ ) and FVR (Fig. $1 \mathrm{~d}, P=0.003, q=0.020$ ) were also observed after the administration of sodium nitroprusside.

In whites, the differences in basal FBF ( $3.6 \pm 1.1$ vs. $4.0 \pm 1.1 \mathrm{ml} / 100 \mathrm{ml}$ tissue $/ \mathrm{min} ; P=0.184$ ) and FVR $(22.7 \pm 1.1$ vs. $20.8 \pm 1.1 \mathrm{mmHg} / \mathrm{ml} / 100 \mathrm{ml}$ tissue/min; $P=0.084)$ between $-653 G$ variant allele carriers and wild-type individuals, respectively, were less substantial than those observed in African Americans and were not statistically significant. In contrast to the 
significant associations observed in African Americans, the - 653A/G genotype was not significantly associated with either FBF or FVR after the administration of either bradykinin or sodium nitroprusside in whites (Fig. 2). Similar results were obtained using a dominant model of inheritance (data not shown).

$-651 \mathrm{G} / \mathrm{A}$

No significant differences in resting $\operatorname{FBF}(P=0.445$ and 0.123$)$ and $\operatorname{FVR}(P=0.340$ and $0.196)$ were observed across the $-651 G / A$ genotype in either African Americans or whites, respectively (see Supplemental Table 2, Supplemental digital content 1, http:// links.lww.com/FPC/A482). Moreover, no significant association between the $-651 G / A$ genotype and the vasodilator response to either bradykinin (FBF: $P=0.119$, FVR: $P=0.225$ ) or sodium nitroprusside (FBF: $P=0.772$, FVR: $P=0.731$ ) was observed in whites (see Supplemental Fig. 1, Supplemental digital content 2, http://links.lww.com/FPC/A483, which shows the forearm vasodilator responses across the $-651 G / A$ genotype). Similar results were observed in African Americans, although only seven - $651 A$ variant allele carriers were identified (see Supplemental Fig. 2, Supplemental digital content 2, http:// links.lww.com/FPC/A483).

$-617 \mathrm{C} / \mathrm{A}$

In whites, $-617 A$ variant allele carriers had lower baseline $\mathrm{FBF}(3.4 \pm 1.1$ vs. $3.9 \pm 1.0 \mathrm{ml} / 100$ $\mathrm{ml}$ tissue/min; $P=0.076)$ and higher FVR $(24.9 \pm 1.1$ vs. $21.0 \pm 1.0 \mathrm{mmHg} / \mathrm{ml} / 100 \mathrm{ml}$ tissue/ $\min ; P=0.035)$ compared with wild-type $(\mathrm{C} / \mathrm{C})$ individuals, respectively; however, only the FVR difference was statistically significant. Vasodilator responses to bradykinin and sodium nitroprusside were also associated with the $-617 C / A$ genotype in whites. Although the difference in FBF did not reach statistical significance (Fig. 3a, $P=0.076$ and Fig. 3c, $P=0.092$, respectively), $-617 A$ variant allele carriers showed significantly higher FVR (Fig. $3 \mathrm{~b}, P=0.030, q=0.120$ and Fig. $3 \mathrm{~d}, P=0.026, q=0.120$, respectively) compared with wild-type individuals. Statistical comparisons were not completed in African Americans as only two$617 A$ variant allele carriers were identified.

\section{Functional significance of the NFE2L2 promoter polymorphisms in vitro}

NFE2L 2 promoter constructs were assessed in transiently transfected HMVEC cells. Wildtype and variant NFE2L2 constructs were used to assess the effects of each variant allele on the functional activity of the promoter under basal conditions and following stimulation with bradykinin, sodium nitroprusside, and hydrogen peroxide. Under basal conditions, HMVEC cells transfected with variant promoter constructs differed significantly from cells transfected with the wild-type promoter construct $(P<0.05$ vs. wild type for all constructs, Fig. 4). Although moderately lower transcriptional activity was observed in vehicle-treated cells bearing the variant $-653 G$ or $-651 A$ constructs ( 27 and $23 \%$ reduction, respectively, compared with the wild type), the most substantial effect was observed with $-617 A$ (42\% reduction compared with the wild type; $P<0.05$ vs. $-653 G$ and $-651 A$ ). Bradykinin, sodium nitroprusside, and hydrogen peroxide each significantly increased the transcriptional activity for all constructs $(P<0.05$ vs. vehicle, Fig. 4). The $-653 G,-651 A$, and $-617 A$ variants each significantly attenuated the activation of luciferase activity irrespective of the stimulus $(P<0.05$ vs. the wild type for all comparisons, Fig. 4$)$.

\section{Discussion}

NRF2 is a key regulator of cardiovascular homeostasis through the suppression of oxidative stress assault [10-13,22,23], which is a major contributor to the development and progression of vascular dysfunction and cardiovascular disease in humans [2,5]. Although polymorphisms in the NFE2L2 gene have recently been associated with oxidative stress- 
related and inflammation-related diseases and drug-induced phenotypes in humans [15,24,25], the impact of NFE2L2 polymorphisms on vascular function has not been studied to date. The current study examined the effects of three polymorphisms ( $-653 A / G,-651 G /$ $A$, and $-617 C / A$ ) within the NFE2L2 promoter region on forearm vasodilator responses to bradykinin (endothelium-dependent) and sodium nitroprusside (endothelium-independent) in healthy African-American and white participants. This study identified a significant association between genetic variants in NFE2L2 and these established peripheral vascular phenotypes [1], which have been shown to correlate with vasodilator responses in coronary arteries and predict the risk of developing cardiovascular disease in humans [5].

Specifically, African-American - $653 G$ variant allele carriers had significantly lower FBF and higher FVR under basal conditions as well as in response to each vasodilator compared with wild-type A/A individuals. Similar associations were observed with the $-617 A$ variant allele in whites, who had lower FBF and significantly higher FVR under basal conditions and in response to each vasodilator compared with wild-type C/ C individuals. Similar genotype-phenotype relationships were observed in response to bradykinin and sodium nitroprusside, suggesting that the mechanism underlying the link between genetic variation in NRF2 and vascular function in humans is endothelial independent.

The results from the transient transfection reporter gene assays show that the $-653 G$,$651 A$, and $-617 A$ variant alleles each reduce NFE2L2 promoter activity in endothelial cells under both basal and stimulated conditions. Consequently, we speculate that these polymorphisms within the NFE2L2 promoter may also be associated with chronically lower vascular NRF2 expression in vivo. Indeed, it is well established that polymorphisms within regulatory regions and enhancer elements of a given gene can exert a profound effect on transcription efficiency and thus alter the levels of mRNA and protein [26]; however, the reporter gene assay experiments do not allow an assessment of whether the variant alleles impact the occurrence of a more physiologically relevant phenotype such as oxidative stress. Moreover, the impact of factors that regulate NFE2L2 at the transcriptional level on NRF2 function in vivo remains unclear. NRF2 is associated with Kelch-like ECH associating protein 1 (KEAP1) and is predominantly localized in the cytoplasm of unstressed cells [8,9]. Oxidative stress disrupts the sequestration of NRF2 by KEAP1 and allows NRF2 translocation to the nucleus, where it regulates the basal and inducible expression of more than 200 known genes [8,9]. In preclinical models, NRF2 deficiency reduces the expression of several antioxidant genes, enhances the expression levels of proinflammatory cytokines, chemokines, and several genes involved in cell proliferation, adhesion, migration, and differentiation, and consequently plays an important role in the regulation of endothelial and vascular smooth muscle cell growth and vascular remodeling [7,12,22]. In addition, NRF2 regulates cardiac remodeling and hypertrophy, and slows the onset of cardiac dysfunction in response to pathological hemodynamic stress [23]. Collectively, these studies suggest that genetic predisposition to chronically lower NRF2 expression in humans, secondary to the presence of polymorphisms within the NFE2L2 promoter that lower transcriptional activity, would be predicted to compromise antioxidant defense systems and result in prolonged oxidative stress, progressive vascular remodeling, and ultimately impaired endotheliumindependent vasodilator responses.

The observed associations between $-653 A / G$ and $-617 C / A$ genotypes and vascular function in African Americans and whites, respectively, were consistent with the in-vitro data and this functional relationship. In contrast, no differences in vasodilator responses were observed in white $-653 G$ variant allele carriers or $-651 A$ variant allele carriers in either racial group. Although the underlying mechanisms remain unclear, the lack of an association between the $-653 A / G$ polymorphism and vasodilator responses in whites could be because of, at least in part, underlying racial differences in vascular function $[18,19]$ and oxidative stress level [27]. It is well established that African Americans have a higher 
prevalence of hypertension and decreased vasodilatory responses to endothelium-dependent or endothelium-independent stimuli compared with white populations [18]. Indeed, racial differences in forearm vasodilator responses were observed in the cohort under investigation $[16,17]$. Predisposition to higher vascular resistance in African Americans leads to increased shear stress on endothelial cells, which activates NAD(P)H oxidase-mediated reactive oxygen species formation in the vasculature [11,23]. Consequently, NAD(P)H oxidase expression is higher and the steady-state balance of nitric oxide/superoxide/peroxynitrite in endothelial cells is shifted in favor of reactive oxygen species in African Americans, which is associated with an impaired vasodilatory capacity [27]. Therefore, we hypothesize that the blunted antioxidant effect associated with the NFE2L2 promoter polymorphisms may be most relevant in African Americans, who are prone to a higher degree of oxidative stress and impaired vascular function compared with white populations. Indeed, this relationship is consistent with our observation that African-American, but not white, $-653 G$ variant allele carriers showed significantly lower FBF and higher FVR at baseline and in response to bradykinin and sodium nitroprusside compared with wild-type individuals despite the modest effects of the $-653 G$ variant on NFE2L2 transcriptional activity in endothelial cells in vitro.

Despite examining the vascular effects of $N R F 2$ polymorphisms in one of the largest cohorts that directly measured vasodilatory responses to infused agonists, the relatively small sample size, particularly in the African Americans studied $(n=64)$, represents a limitation. Although there was a modest degree of power to detect a $30 \%$ difference in FVR in African Americans across the $-653 A / G$ genotype at an $a=0.05$ level $(\beta=0.64)$, only seven $-651 A$ and two $-617 A$ variant allele carriers were identified and these data should be interpreted with caution. In whites, however, there was ample power to detect a $30 \%$ difference in FVR across the $-653 A / G(\beta=0.99),-651 G / A(\beta=0.90)$, and $-617 C / A(\beta=0.92)$ genotypes at an $a=0.05$ level. Therefore, we conclude that a clinically significant association between the $653 \mathrm{~A} / \mathrm{G}$ and $-651 G / A$ genotypes and vasodilator responses does not exist in healthy whites. Although the underlying mechanism remains unclear, the modest effect of the $-653 G$ and $651 A$ variants on NFE2L2 transcription in vitro may not have been large enough to confer an observable phenotype in whites, who are less prone to oxidative stress and vascular dysfunction compared with African-American populations [27]. In contrast, the $-617 A$ variant, which showed the most substantial reduction in luciferase activity in vitro and has been associated previously with acute lung injury in humans [15], was associated with significantly higher FVR in whites. We acknowledge that the lack of in-vivo oxidative stress biomarker measurements in this population, which could have provided a functional insight into the observed polymorphism-specific and race-specific associations between NRF2 and vascular function, is a major limitation of our work. In addition, the current population included healthy volunteers. This could reduce generalization of the observed associations with other populations with enhanced oxidative stress and at a high risk of developing cardiovascular disease, such as individuals with the metabolic syndrome and diabetes, and cigarette smokers. Future studies are warranted to elucidate the contribution of oxidative stress toward the observed NRF2 genotype-vascular function phenotype relationships.

Finally, multiple statistical tests were carried out in this genotype-phenotype analysis, which could potentially lead to false-positive associations. To minimize the impact of multiple comparisons, a false discovery rate $q$-value was calculated for each comparison [20,21]. As all $q$-values were estimated to be less than 0.15 , and the $N F E 2 L 2-653 G$ and $-617 A$ variants have been associated recently with other oxidative stress-related phenotypes in humans [15,24,25], a higher level of confidence exists in the associations reported.

However, it cannot be ruled out that these are simply markers in LD with the true causative loci. Importantly, these associations will ultimately require validation in an independent 
population to confirm the presence of a functional relationship between genetic variants in NFE2L2 and vascular function in humans.

\section{Conclusion}

Genetic variation within the NFE2L2 promoter is associated with impaired forearm vasodilator responses in an endothelial-independent manner, suggesting an important role for NRF2 in the regulation of vascular function in humans. Further studies are necessary to validate the impact of these polymorphisms in the NFE2L2 promoter on NRF2 expression, oxidative stress, vascular function, and the risk of cardiovascular disease in humans.

\section{Supplementary Material}

Refer to Web version on PubMed Central for supplementary material.

\section{Acknowledgments}

The authors gratefully acknowledge Delia Woods, RN, for her nursing assistance and Jeff Petro, BS, for his technical assistance.

This study was supported by funds from the Intramural Research Program of the NIH, National Institute of Environmental Health Sciences to Dr Zeldin (Z01 ES025034) and Dr Kleeberger (Z01 ES100513), grants DK38226, HL65195, and HL060906 to Dr Brown, grant HL085740 to Dr Pretorius, and a Beginning Grant-in-Aid from the American Heart Association to Dr Lee. Its contents are solely the responsibility of the authors and do not necessarily represent the official views of the NIH.

\section{References}

1. Deanfield JE, Halcox JP, Rabelink TJ. Endothelial function and dysfunction: testing and clinical relevance. Circulation. 2007; 115:1285-1295. [PubMed: 17353456]

2. Forstermann U. Nitric oxide and oxidative stress in vascular disease. Pflugers Arch. 2010; 459:923939. [PubMed: 20306272]

3. Yasue H, Matsuyama K, Okumura K, Morikami Y, Ogawa H. Responses of angiographically normal human coronary arteries to intracoronary injection of acetylcholine by age and segment. Possible role of early coronary atherosclerosis. Circulation. 1990; 81:482-490. [PubMed: 2105173]

4. Schachinger V, Britten MB, Zeiher AM. Prognostic impact of coronary vasodilator dysfunction on adverse long-term outcome of coronary heart disease. Circulation. 2000; 101:1899-1906. [PubMed: 10779454]

5. Heitzer T, Schlinzig T, Krohn K, Meinertz T, Munzel T. Endothelial dysfunction, oxidative stress, and risk of cardiovascular events in patients with coronary artery disease. Circulation. 2001; 104:2673-2678. [PubMed: 11723017]

6. Moi P, Chan K, Asunis I, Cao A, Kan YW. Isolation of NF-E2-related factor 2 (Nrf2), a NF-E2-like basic leucine zipper transcriptional activator that binds to the tandem NF-E2/AP1 repeat of the betaglobin locus control region. Proc Natl Acad Sci USA. 1994; 91:9926-9930. [PubMed: 7937919]

7. Leung L, Kwong M, Hou S, Lee C, Chan JY. Deficiency of the Nrf1 and Nrf2 transcription factors results in early embryonic lethality and severe oxidative stress. J Biol Chem. 2003; 278:4802148029. [PubMed: 12968018]

8. Kensler TW, Wakabayashi N, Biswal S. Cell survival responses to environmental stresses via the Keap1-Nrf2-ARE pathway. Annu Rev Pharmacol Toxicol. 2007; 47:89-116. [PubMed: 16968214]

9. Kobayashi M, Yamamoto M. Molecular mechanisms activating the Nrf2-Keap1 pathway of antioxidant gene regulation. Antioxid Redox Signal. 2005; 7:385-394. [PubMed: 15706085]

10. Li J, Ichikawa T, Janicki JS, Cui T. Targeting the Nrf2 pathway against cardiovascular disease. Expert Opin Ther Targets. 2009; 13:785-794. [PubMed: 19530984]

11. Hosoya T, Maruyama A, Kang MI, Kawatani Y, Shibata T, Uchida K, et al. Differential responses of the Nrf2-Keap1 system to laminar and oscillatory shear stresses in endothelial cells. J Biol Chem. 2005; 280:27244-27250. [PubMed: 15917255] 
12. Chen XL, Dodd G, Thomas S, Zhang X, Wasserman MA, Rovin BH, et al. Activation of Nrf2/ ARE pathway protects endothelial cells from oxidant injury and inhibits inflammatory gene expression. Am J Physiol Heart Circ Physiol. 2006; 290:H1862-H1870. [PubMed: 16339837]

13. Xue M, Qian Q, Adaikalakoteswari A, Rabbani N, Babaei-Jadidi R, Thornalley PJ. Activation of NF-E2-related factor-2 reverses biochemical dysfunction of endothelial cells induced by hyperglycemia linked to vascular disease. Diabetes. 2008; 57:2809-2817. [PubMed: 18633117]

14. Jyrkkanen HK, Kansanen E, Inkala M, Kivela AM, Hurttila H, Heinonen SE, et al. Nrf2 regulates antioxidant gene expression evoked by oxidized phospholipids in endothelial cells and murine arteries in vivo. Circ Res. 2008; 103:e1-e9. [PubMed: 18535259]

15. Marzec JM, Christie JD, Reddy SP, Jedlicka AE, Vuong H, Lanken PN, et al. Functional polymorphisms in the transcription factor NRF2 in humans increase the risk of acute lung injury. FASEB J. 2007; 21:2237-2246. [PubMed: 17384144]

16. Pretorius MM, Gainer JV, Van Guilder GP, Coelho EB, Luther JM, Fong P, et al. The bradykinin type 2 receptor BE1 polymorphism and ethnicity influence systolic blood pressure and vascular resistance. Clin Pharmacol Ther. 2008; 83:122-129. [PubMed: 17522594]

17. Lee CR, Pretorius M, Schuck RN, Burch LH, Bartlett J, Williams SM, et al. Genetic variation in soluble epoxide hydrolase (EPHX2) is associated with forearm vasodilator responses in humans. Hypertension. 2011; 57:116-122. [PubMed: 21098312]

18. Stein CM, Lang CC, Nelson R, Brown M, Wood AJ. Vasodilation in black Americans: attenuated nitric oxide-mediated responses. Clin Pharmacol Ther. 1997; 62:436-443. [PubMed: 9357395]

19. Cardillo C, Kilcoyne CM, Cannon RO 3rd, Panza JA. Attenuation of cyclic nucleotide-mediated smooth muscle relaxation in blacks as a cause of racial differences in vasodilator function. Circulation. 1999; 99:90-95. [PubMed: 9884384]

20. Storey JD, Tibshirani R. Statistical significance for genomewide studies. Proc Natl Acad Sci USA. 2003; 100:9440-9445. [PubMed: 12883005]

21. Gauderman WJ. Candidate gene association analysis for a quantitative trait, using parent-offspring trios. Genet Epidemiol. 2003; 25:327-338. [PubMed: 14639702]

22. Levonen AL, Inkala M, Heikura T, Jauhiainen S, Jyrkkanen HK, Kansanen E, et al. Nrf2 gene transfer induces antioxidant enzymes and suppresses smooth muscle cell growth in vitro and reduces oxidative stress in rabbit aorta in vivo. Arterioscler Thromb Vasc Biol. 2007; 27:741-747. [PubMed: 17255530]

23. Li J, Ichikawa T, Villacorta L, Janicki JS, Brower GL, Yamamoto M, et al. Nrf2 protects against maladaptive cardiac responses to hemodynamic stress. Arterioscler Thromb Vasc Biol. 2009; 29:1843-1850. [PubMed: 19592468]

24. Cordova E, Velazquez-Cruz R, Centeno F, Baca V, Orozco L. The NRF2 gene variant, - 653G/A, is associated with nephritis in childhood-onset systemic lupus erythematosus. Lupus. 2010; 19:1237-1242. [PubMed: 20507872]

25. Bouligand J, Cabaret O, Canonico M, Verstuyft C, Dubert L, Becquemont L, et al. Estrogen and Thromboembolism Risk (ESTHER) Study Group. Effect of NFE2L2 genetic polymorphism on the association between oral estrogen therapy and the risk of venous thromboembolism in postmenopausal women. Clin Pharmacol Ther. 2011; 89:60-64. [PubMed: 21107315]

26. Wray GA, Hahn MW, Abouheif E, Balhoff JP, Pizer M, Rockman MV, et al. The evolution of transcriptional regulation in eukaryotes. Mol Biol Evol. 2003; 20:1377-1419. [PubMed: 12777501]

27. Kalinowski L, Dobrucki IT, Malinski T. Race-specific differences in endothelial function: predisposition of African Americans to vascular diseases. Circulation. 2004; 109:2511-2517. [PubMed: 15159296] 

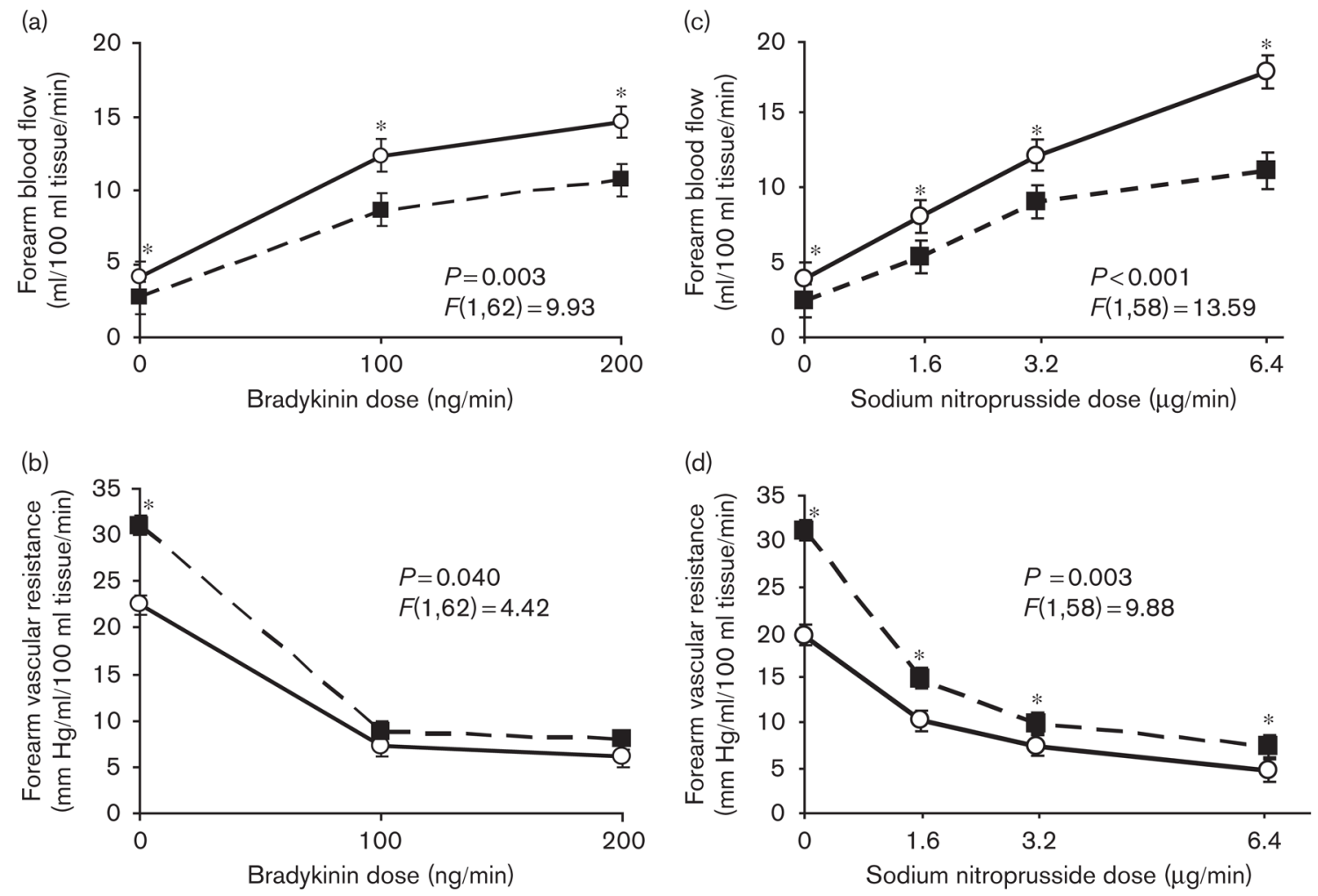

\section{$A / A-\square-A / G+G / G$}

Fig. 1.

$N F E 2 L 2$ - 653A/G genotype and vasodilator responses in African Americans. Forearm blood flow $(\mathrm{a}, \mathrm{c})$ and forearm vascular resistance $(\mathrm{b}, \mathrm{d})$ at baseline and upon the administration of bradykinin $(a, b)$ or sodium nitroprusside $(c, d)$ were determined using a dominant model of inheritance. Results are presented as mean \pm SEM. The repeated-measures ANOVA $P$ and $F$ values for each comparison are provided. * Post hoc $(P<0.05)$ versus wildtype (A/A). ANOVA, analysis of variance. 

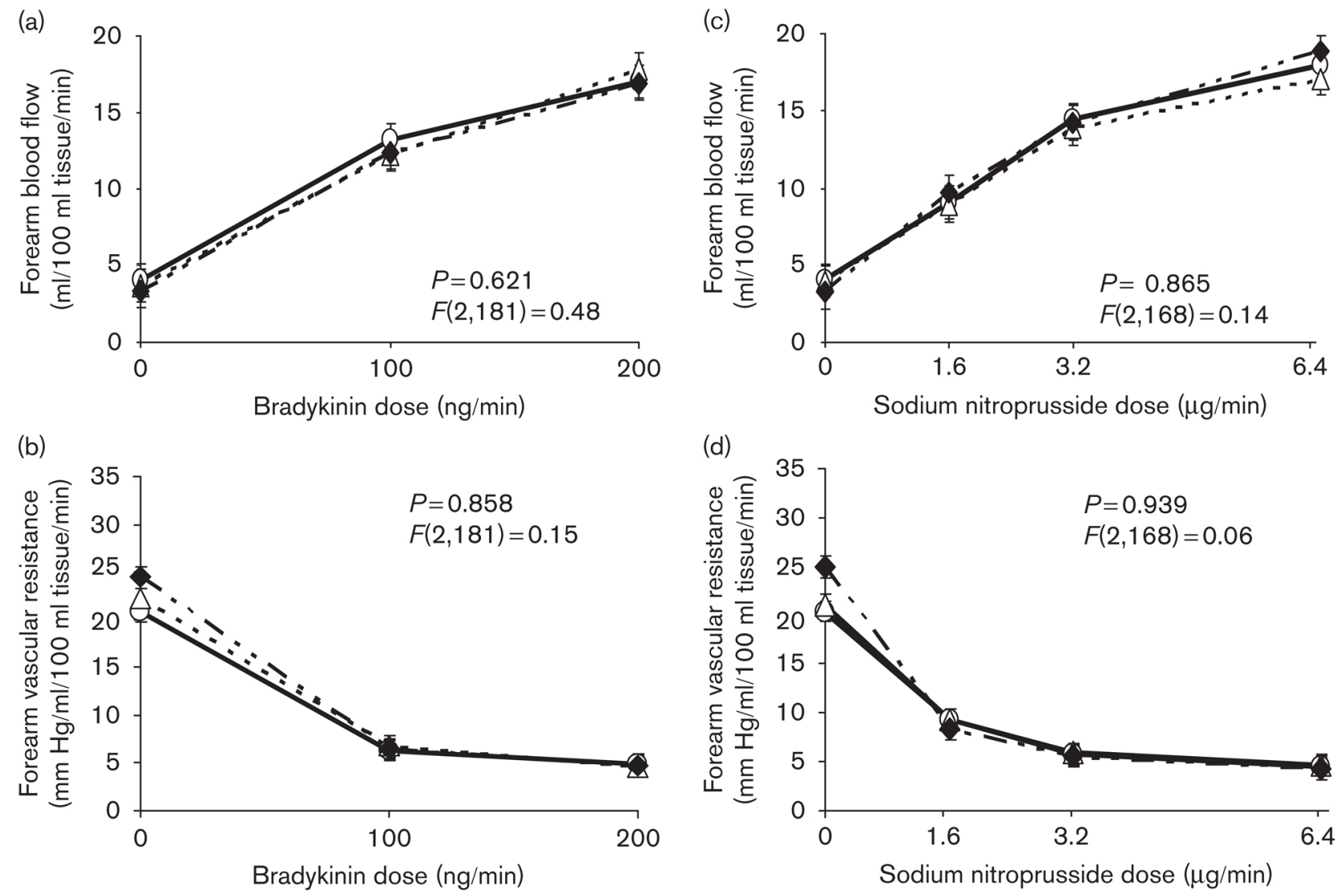

\section{$\longrightarrow \mathrm{O}=\mathrm{A} / \mathrm{A} \quad \cdots \Delta \cdot \cdots \mathrm{A} / \mathrm{G} \longrightarrow \bullet \mathrm{G} / \mathrm{G}$}

Fig. 2.

$N F E 2 L 2-653 A / G$ genotype and vasodilator responses in whites. Forearm blood flow (a, c) and forearm vascular resistance $(b, d)$ at baseline and upon the administration of bradykinin $(\mathrm{a}, \mathrm{b})$ or sodium nitroprusside $(\mathrm{c}, \mathrm{d})$ were determined using an additive model of inheritance. Results are presented as mean \pm SEM. The repeated-measures ANOVA $P$ and $F$ values for each comparison are provided. * Post hoc $(P<0.05)$ versus wild-type $(\mathrm{A} / \mathrm{A})$. ANOVA, analysis of variance. 

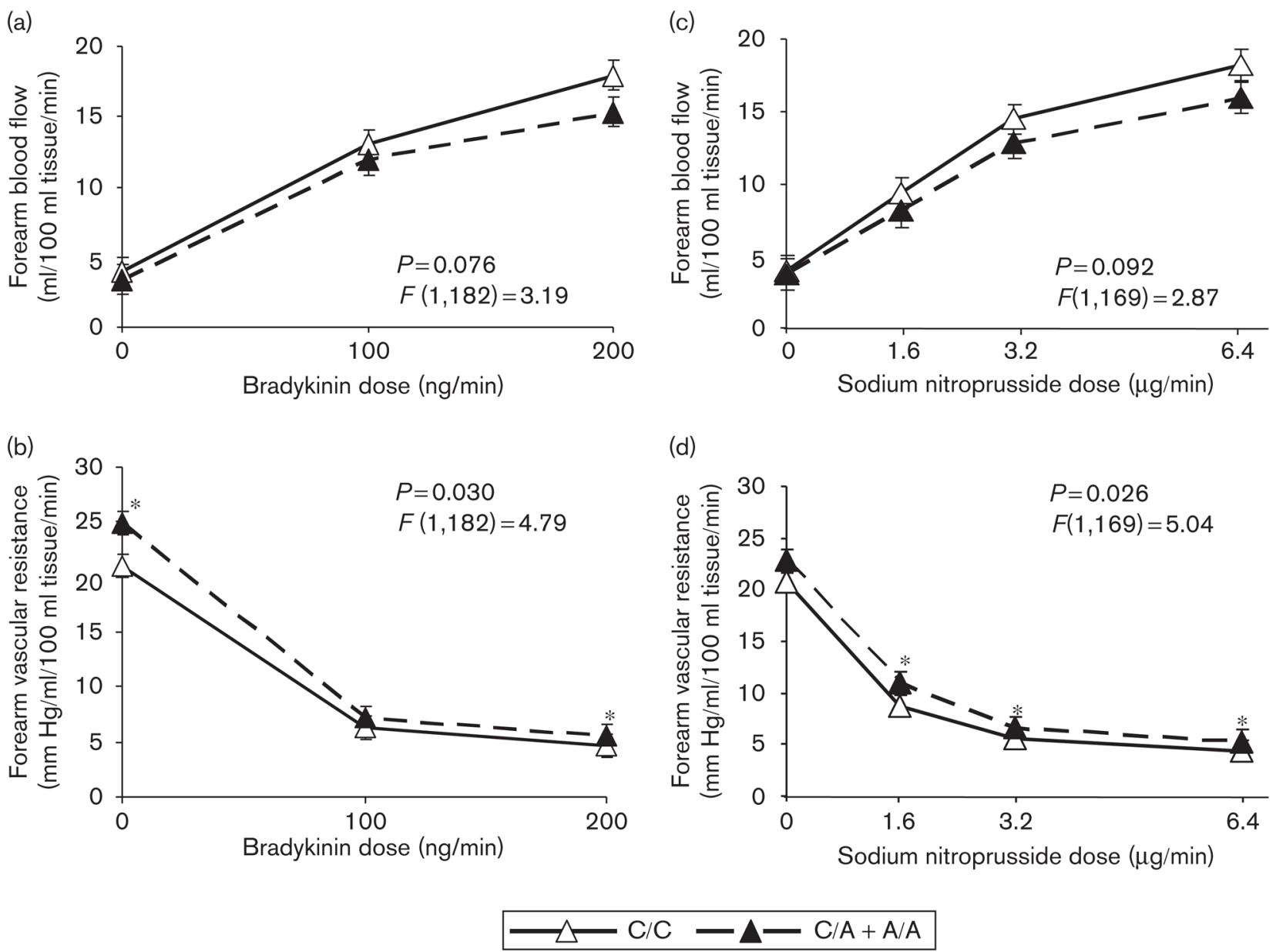

Fig. 3.

NFE2L2 - 617C/A genotype and vasodilator responses in whites. Forearm blood flow (a, c) and forearm vascular resistance $(b, d)$ at baseline and upon the administration of bradykinin $(\mathrm{a}, \mathrm{b})$ or sodium nitroprusside $(\mathrm{c}, \mathrm{d})$ were determined using a dominant model of inheritance. Results are presented as mean \pm SEM. The repeated-measures ANOVA $P$ and $F$ values for each comparison are provided. * Post hoc $(P<0.05)$ versus wild-type $(\mathrm{C} / \mathrm{C})$. ANOVA, analysis of variance. 


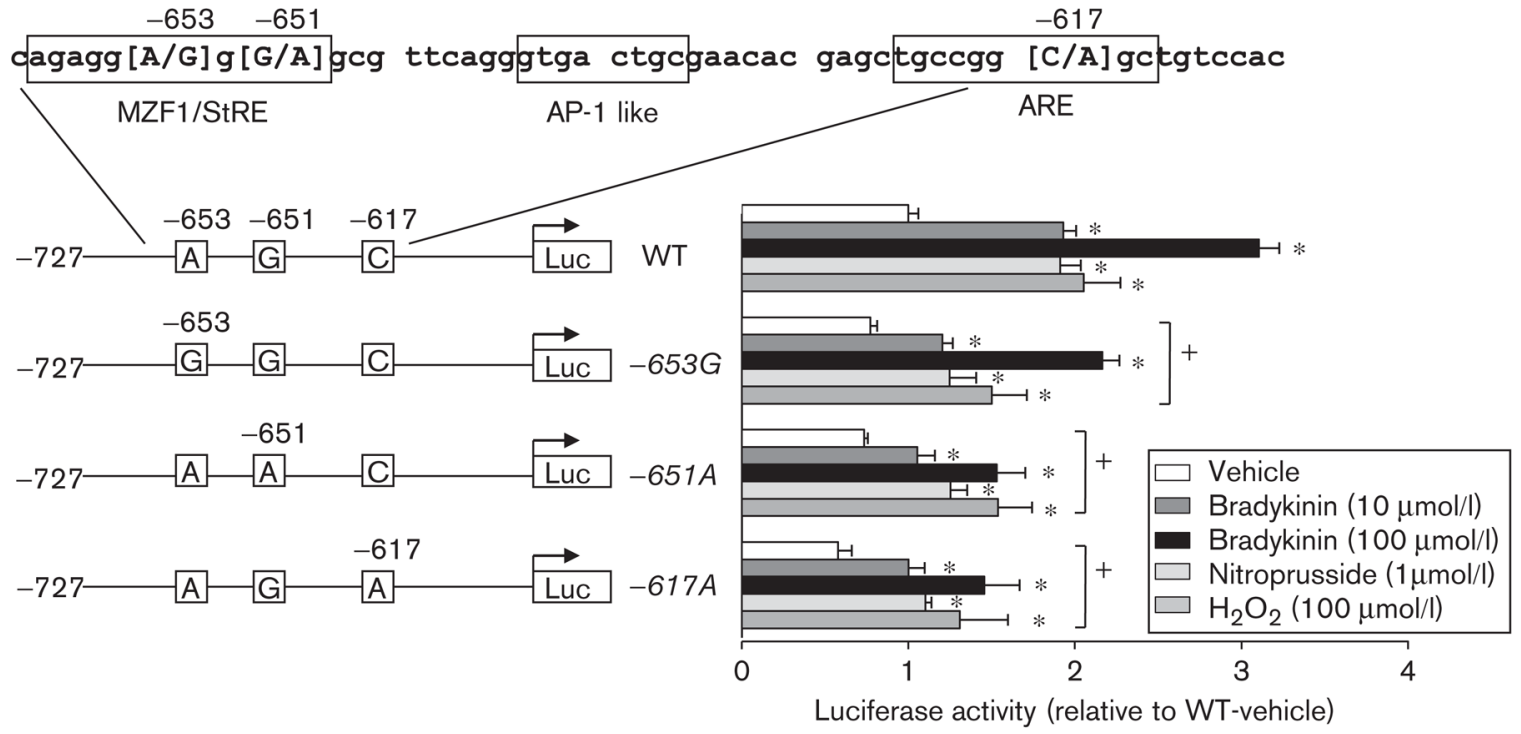

Fig. 4.

Functional significance of the NFE2L2 promoter polymorphisms in vitro. The relative luciferase activity of $N F E 2 L 2$ promoter constructs after exposure to vehicle (basal conditions), bradykinin $(0.01$ or $0.1 \mathrm{mmol} / \mathrm{l})$, sodium nitroprusside $(0.1 \mathrm{mmol} / \mathrm{l})$, or hydrogen peroxide $(0.1 \mathrm{mmol} / \mathrm{l})$ was compared following transient transfection in human microvascular endothelial cells (HMVEC). The sequence identifies the three polymorphisms studied $(-653 A / G,-651 A / G$, and $-617 C / A)$ and putative transcription factor-binding sites including the myeloid zinc finger-1 (MZF1)/stress response element (StRE), an AP-1-like sequence, and the antioxidant response element (ARE). Luciferase activity was expressed relative to the wild-type (WT) vehicle group. *Post hoc $(P<0.05)$ compared with the vehicle (within each genotype group); ${ }^{+}$Post hoc $(P<0.05)$ compared with WT (within each treatment group). 


\section{Table 1}

Participant characteristics by race

\begin{tabular}{lcc}
\hline Variable & African Americans & Whites \\
\hline$N$ & 64 & 184 \\
Age (years) & $32.9 \pm 1.3$ & $31.6 \pm 0.8$ \\
Females $(\%)$ & $39(60.9 \%)$ & $80(43.5 \%)^{*}$ \\
BMI $\left(\mathrm{kg} / \mathrm{m}^{2}\right)$ & $26.1 \pm 0.4$ & $24.6 \pm 0.3 *$ \\
Smoker $(\%$ yes) & $4(6.3 \%)$ & $19(10.3 \%)$ \\
MAP $(\mathrm{mmHg})$ & $87.3 \pm 1.2$ & $83.5 \pm 0.7^{*}$ \\
HR $(\mathrm{bpm})$ & $64 \pm 1.4$ & $65 \pm 0.8$ \\
FBF $(\mathrm{ml} / 100 \mathrm{ml} \mathrm{tissue} / \mathrm{min})$ & $3.6 \pm 1.1$ & $3.8 \pm 1.0$ \\
FVR $(\mathrm{mmHg} / \mathrm{ml} / 100 \mathrm{ml}$ tissue/min) & $25.2 \pm 1.1$ & $21.8 \pm 1.0 *$ \\
\hline
\end{tabular}

Data are presented as mean \pm SEM or count (proportion).

FBF, baseline forearm blood flow; FVR, baseline forearm vascular resistance; HR, heart rate; MAP, mean arterial pressure. * $P<0.05$ versus African Americans. 
